\title{
Irradiation of mixed ices as a laboratory cometary model
}

\author{
Maria Colin-Garcia ${ }^{1}$, Alicia Negrón-Mendoza ${ }^{1}$, \\ Sergio Ramos-Bernal ${ }^{1}$, and Elizabeth Chacon ${ }^{2}$ \\ ${ }^{1}$ Instituto de Ciencias Nucleares, Universidad Nacional Autónoma de México, C. U. Mexico, \\ D. F. Mexico 04510 \\ ${ }^{2}$ Facultad de Ciencias de la Tierra, Universidad Autónoma de Nuevo León \\ email: negron@nucleares .unam.mx
}

\begin{abstract}
Icy bodies in space are being irradiated continuously by ionizing radiation. Therefore, the transformation of organic molecules trapped in extraterrestrial ices might have been possible. This work studied a bulk irradiation of a mixture of some constituents of cometary nuclei. The results show that the formation of different compounds, among them ammonia, carbon dioxide, amines, ureas, free amino acids, and oligomeric material, yields carboxylic acids, amino acids, and purines upon hydrolysis.
\end{abstract}

Keywords. Astrobiology, astrochemistry, molecular processes, laboratory, comets

\section{Introduction}

Comets are minor bodies in our Solar System as old as the Solar System itself. They were thought to provide unique information about the pristine Solar System. However, chemical evolution processes that occurred at early stages of the Solar System and after could have transformed the organics within them. Irradiation of those bodies has occurred since they were formed from interstellar grains; UV photons and cosmic ions had already interacted with those grains (Hudson \& Moore 1999). Later, during their storage in the reservoirs mentioned, their surfaces were continuously irradiated by cosmic rays. All of this exposure alters the chemical and physical properties of those bodies (Hudson \& Moore 1999).

Comet nuclei are composed of rock, dust, water ice, and frozen gases such as carbon monoxide, carbon dioxide, methane, and ammonia. Also there are organic molecules such as methanol, hydrogen cyanide, formaldehyde, ethanol, and ethane, as well as perhaps more complex molecules such as long-chain hydrocarbons and amino acids.

The aim of this work is to give another contribution to the understanding of the evolution of the organics in comets, studying the products obtained by the behavior of an (aqueous-dominant) simplified cometary model exposed to high doses of ionizing radiation. The experiment was performed using gamma rays of the same LET (linear energy transfer) of the most abundant protons in the cosmic rays (Draganic et al. 1984). The mixture consisted of $\mathrm{HCN} / \mathrm{CH}_{3} \mathrm{OH} / \mathrm{CH}_{3} \mathrm{CN} / \mathrm{C}_{2} \mathrm{H}_{5} \mathrm{CN} / \mathrm{HCOOH}$, in a proportion in which they appeared in a dense interstellar cloud (1: 0.6: $0.2: 0.1: 0.05)$. The samples were at $298 \mathrm{~K}$ and $77 \mathrm{~K}$. 


\section{Experimental}

Irradiations were carried out using a gamma ray source of ${ }^{60} \mathrm{Co}$ (Gamma-beam 651PT) at ICN, UNAM. Frozen and liquid solutions were exposed to different radiation doses from 3 to $419 \mathrm{kGy}$. After irradiation, the samples were melted and the following measurements were made directly on the irradiated samples: determination of urea, $\mathrm{pH}$ of the irradiated solution, amino acids, and the non-reacted fraction of the reactants (ColínGarcía et al. 2008). For non-volatile compounds the bulk of irradiated solution (300 ml) was lyophilized. After this procedure, the dry residue was weighted and analyzed by IR spectroscopy and HPLC. An aliquot of the dry residue $(50 \mathrm{mg})$ was hydrolyzed with hydrochloric acid diluted in methanol, 1: 5. Then, samples were esterified and analyzed by gas chromatography (Colín-García et al. 2008).

\section{Results and discussion}

The radiolysis of the multi-component system is a composite process that can be considered roughly as the sum of the behavior of the individual constituents of the system. Cross-reactions may occur. Ionizing radiation induced an abundant formation of a dark-colored mixture of non-volatile compounds. The inventory of radiolytical products includes gases, amines, amino acids, carboxylic acids, and oligomeric material. There is formation and accumulation of radiolytic products. At increasing doses, the primary chemical constituents of the model system become depleted, while the radiolytic products, once formed, accumulate.

The amount and aspect of the dry residue depend on the dose received and the temperature of irradiation. In both sets of experiments the dry residue increased with dose.

The nature of the oligomers is complex, mainly consisting of polyesters and polyamides with urea fragments. Carboxylic acids were detected in the hydrolyzed material. The products detected at $77 \mathrm{~K}$ were malonic, succinic, glutaric, carboxysuccinic, citric, and tricarballylic acids. Free amino acids are produced in very small quantities. The production of these compounds is also dose and temperature dependant.

\section{Final Remarks}

The oligomer is produced by addition or abstraction reactions of the radicals from water ices radiolysis and $\mathrm{CN}$-containing molecules. The radiochemical yields in ice were one or two orders of magnitude lower. This is due mainly to the rigid structure of the ice and the limited mobility of the radicals at low temperature. The results found in this study underline the importance of radiation-induced reactions as an energy source in extraterrestrial scenarios like comets or other icy objects.

\section{Acknowledgements}

This work was supported by DGAPA-UNAM Grant IN223406. The support of the Posgrado en Ciencias Biológicas, UNAM for one of us (MC) is acknowledged.

\section{References}

Colín-García, M., Negrón-Mendoza, A., \& Ramos-Bernal, S. 2008, Astrobiology, in press

Draganič, I. G., Draganič, Z. D., \& Vujosevič, S. 1984, Icarus, 60, 464

Hudson, R. L. \& Moore, M. H. 1999, Icarus, 140, 450 